\title{
SECOND ORDER NECESSARY CONDITIONS OF OPTIMALITY FOR STOCHASTIC SYSTEMS WITH VARIABLE DELAY
}

UDC 519.21

\author{
CH. A. AGAYEVA
}

\begin{abstract}
The purpose of this paper is to give necessary conditions of optimality of nonlinear stochastic control systems with variable delay for singular controls. As a result, the second order necessary optimality condition for the stochastic system with uncontrolled diffusion coefficient is obtained.
\end{abstract}

\section{INTRODUCTION}

Stochastic differential equations with delay have found many applications in automatic control theory, in the theory of self-oscillating systems, etc., where real systems are exposed to the influences of random disturbances, which cannot be ignored [1, 2]. The necessary conditions of optimality for deterministic control problems are obtained in 3 . The first and second order necessary conditions of optimality for the stochastic control systems without delay are proved in [4. Optimal control problems for the systems described by means of stochastic differential equations with delay have already been investigated and the first order necessary condition of optimality for the stochastic system is obtained in the research [5, 6]. This research is dedicated to the problem of stochastic optimal control with variable delay under the influence of singular controls.

\section{Statement of the Problem}

Throughout this paper, unless otherwise specified, we let $(\Omega, F, \mathrm{P})$ be a complete probability space with the filtration $\left\{F^{t}: t_{0} \leq t \leq t_{1}\right\}$ generated by the Wiener process $w_{t}$ and $F^{t}=\sigma\left(w_{s} ; t_{0} \leq s \leq t\right)$. Let $\mathbf{R}^{m}$ denote the $m$-dimensional real vector space and $|\cdot|$ denote the Euclidean norm in $\mathbf{R}^{m}$. Let $\mathrm{E}$ represent the expectation. Let $L_{F}^{2}\left(t_{0}, t_{1}, \mathbf{R}^{n}\right)$ denote the space of predictable processes $x_{t}(\omega)$ such that $\mathrm{E} \int_{t_{0}}^{t_{1}}\left|x_{t}\right|^{2} d t<+\infty$. If $A$ is a vector or a matrix, its transpose is denoted by $A^{*}$.

Consider the following stochastic system with variable delay on state:

$$
\begin{gathered}
d x_{t}=g\left(x_{t}, x_{t-h(t)}, u_{t}, t\right) d t+f\left(x_{t}, x_{t-h(t)}, t\right) d w_{t}, \quad t \in\left(t_{0}, t_{1}\right], \\
x_{t_{0}}=x_{0}, \\
x_{t}=\Phi(t), \quad t \in\left[t_{0}-h\left(t_{0}\right), t_{0}\right), \\
u_{t}(\omega) \in U_{\partial} \equiv\left\{u .(\cdot) \in L_{F}^{2}\left(t_{0}, t_{1} ; \mathbf{R}^{m}\right) \mid u .(\omega) \in U \subset \mathbf{R}^{m} \text { a.c. }\right\},
\end{gathered}
$$

2010 Mathematics Subject Classification. Primary 93E20, 49K45.

Key words and phrases. Stochastic differential equations with delay, stochastic control problem, necessary condition of optimality, singular controls, adjoint stochastic differential equations. 
where $U$ is a nonempty bounded set and $\Phi(t)$ is a piecewise continuous nonrandom function. In addition to that, $h(t) \geq 0$ is a continuously differentiable, nonrandom function such that $d h(t) / d t<1$. Assume that it is required to minimize the functional:

$$
J(u)=\mathrm{E}\left\{p\left(x_{t_{1}}\right)+\int_{t_{0}}^{t_{1}} l\left(x_{t}, u_{t}, t\right) d t\right\},
$$

in the set of admissible controls $U_{\partial}$. The pair $\left(x_{t}^{0}, u_{t}^{0}\right)$, which is the solution of problem (1)-(5), will be called optimal. Assume that the following requirements are satisfied:

I. Functions $l, g, f$ are continuous with respect to total arguments:

$$
\begin{gathered}
l(x, u, t): \mathbf{R}^{n} \times \mathbf{R}^{m} \times\left[t_{0}, t_{1}\right] \rightarrow \mathbf{R}^{1} ; \\
g(x, y, u, t): \mathbf{R}^{n} \times \mathbf{R}^{n} \times \mathbf{R}^{m} \times\left[t_{0}, t_{1}\right] \rightarrow \mathbf{R}^{n} ; \\
f(x, y, t): \mathbf{R}^{n} \times \mathbf{R}^{n} \times\left[t_{0}, t_{1}\right] \rightarrow \mathbf{R}^{n \times n} .
\end{gathered}
$$

II. When $t, u$ are fixed, then the functions $l, g, f$ satisfy the conditions:

$$
\begin{gathered}
(1+|x|+|y|)^{-1}\left(|g(x, y, u, t)|+\left|g_{x}(x, y, u, t)\right|\right. \\
\left.+\left|g_{y}(x, y, u, t)\right|+|f(x, y, t)|+\left|f_{x}(x, y, t)\right|+\left|f_{y}(x, y, t)\right|\right) \leq N \\
(1+|x|)^{-1}\left(|l(x, u, t)|+\left|l_{x}(x, u, t)\right|\right) \leq N .
\end{gathered}
$$

III. Function $p(x): \mathbf{R}^{n} \rightarrow \mathbf{R}^{1}$ is continuously differentiable and

$$
|p(x)|+\left|p_{x}(x)\right| \leq N(1+|x|) .
$$

The notation $y_{t}=x_{t-h(t)}$ will be used throughout this paper. The following necessary condition of optimality for the stochastic control problem (11) - (5) in terms of Pontryagin's maximum principle is obtained in [5].

Theorem 2.1. Let conditions $I-I I I$ hold, where $\left(x_{t}^{0}, u_{t}^{0}\right)$ is an optimal solution of the problem (1) -(5) and the random processes $\left(\psi_{t}, \beta_{t}\right) \in L_{F}^{2}\left(t_{0}, t_{1} ; \mathbf{R}^{n}\right) \times L_{F}^{2}\left(t_{0}, t_{1} ; \mathbf{R}^{n \times n}\right)$ are the solutions of the following adjoint equations:

$$
\begin{aligned}
d \psi_{t} & =-\left[H_{x}\left(\psi_{t}, x_{t}^{0}, y_{t}^{0}, u_{t}^{0}, t\right)+\left.H_{y}\left(\psi_{z}, x_{z}^{0}, y_{z}^{0}, u_{z}^{0}, z\right)\right|_{z=s(t)} s^{\prime}(t)\right] d t+\beta_{t} d w_{t}, \\
& t_{0} \leq t<t_{1}-h\left(t_{1}\right), \\
d \psi_{t} & =-H_{x}\left(\psi_{t}, x_{t}^{0}, y_{t}^{0}, u_{t}^{0}, t\right)+\beta_{t} d w_{t}, \quad t_{1}-h\left(t_{1}\right) \leq t<t_{1}, \\
\psi_{t_{1}} & =-p_{x}\left(x_{t_{1}}^{0}\right)
\end{aligned}
$$

where $t=s(\tau)$ is a solution of the equation $\tau=t-h(t)$. Then for all $u \in U$ a.e. in $\left[t_{0}, t_{1}\right)$ the following maximum principle holds:

$$
\max _{u \in U} H\left(\psi_{t}, x_{t}^{0}, y_{t}^{0}, u, t\right)=H\left(\psi_{t}, x_{t}^{0}, y_{t}^{0}, u^{0}, t\right), \quad \text { a.c.; }
$$

here,

$$
H\left(\psi_{t}, x_{t}, y_{t}, u_{t}, t\right)=\psi_{t}^{*} g\left(x_{t}, y_{t}, u_{t}, t\right)+\beta_{t}^{*} f\left(x_{t}, y_{t}, t\right)-l\left(x_{t}, u_{t}, t\right)
$$


Let $\bar{u}_{t}=u_{t}^{0}+\Delta u_{t}$ be some admissible control and $\bar{x}_{t}=x_{t}^{0}+\Delta x_{t}$ the corresponding trajectory of system (1)-(4). We will use the following identities:

$$
\begin{aligned}
& d \Delta x_{t}= {\left[g\left(\bar{x}_{t}, \bar{y}_{t}, \bar{u}_{t}, t\right)-g\left(x_{t}^{0}, y_{t}^{0}, u_{t}^{0}, t\right)\right] d t+\left[f\left(\bar{x}_{t}, \bar{y}_{t}, t\right)-f\left(x_{t}^{0}, y_{t}^{0}, t\right)\right] d w_{t} } \\
&=\left\{\Delta_{\bar{u}} g\left(x_{t}^{0}, y_{t}^{0}, u_{t}^{0}, t\right)+g_{x}\left(x_{t}^{0}, y_{t}^{0}, u_{t}^{0}, \nu_{t}^{0}, t\right) \Delta x_{t}+g_{y}\left(x_{t}^{0}, y_{t}^{0}, u_{t}^{0}, \nu_{t}^{0}, t\right) \Delta y_{t}\right\} d t \\
&+\left\{f_{x}\left(x_{t}^{0}, y_{t}^{0}, t\right) \Delta x_{t}+f_{y}\left(x_{t}^{0}, y_{t}^{0}, t\right) \Delta x_{t}\right\} d w_{t}+\eta_{t}, \quad t \in\left(t_{0}, t_{1}\right], \\
& \Delta x_{t}=0, \quad t \in\left[t_{0}-h\left(t_{0}\right), t_{0}\right],
\end{aligned}
$$

where

$$
\begin{aligned}
\eta_{t}=\left\{\int_{0}^{1}\left[g_{x}^{*}\left(x_{t}^{0}+\mu \Delta x_{t}, \bar{y}_{t}, \bar{u}_{t}, t\right)-g_{x}^{*}\left(x_{t}^{0}, y_{t}^{0}, u_{t}^{0}, t\right)\right] \Delta x_{t} d \mu\right. \\
\left.\quad+\int_{0}^{1}\left[g_{y}^{*}\left(x_{t}^{0}, y_{t}^{0}+\mu \Delta y_{t}, \bar{u}_{t}, t\right)-g_{y}^{*}\left(x_{t}^{0}, y_{t}^{0}, u_{t}^{0}, t\right)\right] \Delta y_{t} d \mu\right\} d t \\
+\left\{\int_{0}^{1}\left[f_{x}^{*}\left(x_{t}^{0}+\mu \Delta x_{t}, \bar{y}_{t}, t\right)-f_{x}^{*}\left(x_{t}^{0}, \bar{y}_{t}, t\right)\right] \Delta x_{t} d \mu\right. \\
\left.+\int_{0}^{1}\left[f_{y}^{*}\left(x_{t}^{0}, y_{t}^{0}+\mu \Delta y_{t}, t\right)-f_{y}^{*}\left(x_{t}^{0}, y_{t}^{0}, t\right)\right] \Delta y_{t} d \mu\right\} d w_{t} .
\end{aligned}
$$

We need the following lemma.

Lemma 2.1. A solution of equation (8) may be described as follows:

$$
\Delta x_{t}=\int_{t_{0}}^{t} Q_{t \tau}\left[\Delta_{\bar{u}} g\left(x_{\tau}^{0}, y_{\tau}^{0}, u_{\tau}^{0}, \tau\right)+\eta_{\tau}\right] d \tau
$$

where $Q_{t \tau}$ is a solution of

$$
\begin{aligned}
d Q_{t \tau}= & \left(g_{x}\left(x_{t}^{0}, y_{t}^{0}, u_{t}^{0}, t\right) Q_{t \tau}+g_{y}\left(x_{t}^{0}, y_{t}^{0}, u_{t}^{0}, t\right) Q_{r(t) \tau}\right) d t \\
& \quad+\left(f_{x}\left(x_{t}^{0}, y_{t}^{0}, t\right) Q_{t \tau}+f_{y}\left(x_{t}^{0}, y_{t}^{0}, t\right) Q_{r(t) \tau}\right) d w_{t}, \quad t>\tau ; \\
Q_{\tau \tau}= & I ; \quad t<\tau ; \\
Q_{t \tau}= & 0, \quad t
\end{aligned}
$$

here $r(t)=t-h(t)$.

Proof. Differentiating expression (9) we have:

$$
\begin{aligned}
d \Delta x_{t}= & \Delta_{\bar{u}} g\left(x_{t}^{0}, y_{t}^{0}, u_{t}^{0}, t\right)+\eta_{t} \\
& +\int_{t_{0}}^{t} d Q_{t \tau} \times\left[\Delta_{\bar{u}} g\left(x_{\tau}^{0}, y_{\tau}^{0}, u_{\tau}^{0}, t\right)+\eta_{\tau}\right] d \tau .
\end{aligned}
$$


By using (10) we obtain the following:

$$
\begin{aligned}
d \Delta x_{t}= & \Delta_{\bar{u}} g\left(x_{t}^{0}, y_{t}^{0}, u_{t}^{0}, t\right)+\eta_{t} \\
& +\int_{t_{0}}^{t}\left\{\left(g_{x}\left(x_{t}^{0}, y_{t}^{0}, u_{t}^{0}, t\right) Q_{t \tau}+g_{y}\left(x_{t}^{0}, y_{t}^{0}, u_{t}^{0}, t\right) Q_{r(t) \tau}\right) d t\right. \\
& \left.\quad+\left(f_{x}\left(x_{t}^{0}, y_{t}^{0}, t\right) Q_{t \tau}+f_{y}\left(x_{t}^{0}, y_{t}^{0}, t\right) Q_{r(t) \tau}\right) d w_{t}\right\} \\
& \times\left[\Delta_{\bar{u}} g\left(x_{\tau}^{0}, y_{\tau}^{0}, u_{\tau}^{0}, t\right)+\eta_{\tau}\right] d \tau .
\end{aligned}
$$

Then with the help of some simple transformations we obtain that $\Delta x_{t}$ satisfies equation (9). The lemma is proved.

We shall use the following definition.

Definition 2.1. An admissible control $u_{t}(\omega), t \in\left[t_{0}, t_{1}\right]$, is said to be singular in stochastic optimal control problems if there exists a subset $\bar{U} \subset U$, such that for all $\nu_{t}(\omega) \in \bar{U}$, $t \in\left[t_{0}, t_{1}\right]$ with probability one, the following relation holds:

$$
\Delta_{\nu} H\left(\psi_{t}, x_{t}, x_{t-h(t)}, u_{t}, t\right) \equiv 0
$$

Here

$$
\Delta_{\nu} H\left(\psi_{t}, x_{t}, x_{t-h(t)}, u_{t}, t\right) \equiv H\left(\psi_{t}, x_{t}, x_{t-h(t)}, \nu, t\right)-H\left(\psi_{t}, x_{t}, x_{t-h(t)}, u_{t}, t\right) .
$$

It is clear that when condition (11) holds, the maximum principle does not apply, and in this particular case it is impossible to determine the optimal control using (17). Therefore we must have a necessary condition of optimality for singular controls.

Now, suppose that the following requirements are satisfied:

A1. Functions $l, g, f$ and their derivatives are continuous in $(x, u, t)$ and have no more than a linear growth.

A2. Functions $l, g, f$ are twice continuously differentiable with respect to $(x, y)$, and their derivatives are bounded.

A3. Function

$$
p(x): \mathbf{R}^{n} \rightarrow \mathbf{R}^{1}
$$

is twice continuously differentiable and

$$
|p(x)|+\left|p_{x}(x)\right| \leq N(1+|x|), \quad\left|p_{x x}\right| \leq N .
$$

Theorem 2.2. Let conditions A1-A3 hold, where $u_{t}^{0}$ is a singular optimal control of problem (11) -(5) and the random processes

$$
\left(\psi_{t}, \beta_{t}\right) \in L_{F}^{2}\left(t_{0}, t_{1} ; \mathbf{R}^{n}\right) \times L_{F}^{2}\left(t_{0}, t_{1} ; \mathbf{R}^{n \times n}\right)
$$

are the solutions of the system (6). Then for all $\nu \in \bar{U}$, we have the following:

$$
\begin{aligned}
\mathrm{E} \Delta_{\nu} g^{*} & \left(x_{\tau}^{0}, y_{\tau}^{0}, u_{\tau}^{0}, \tau\right) K(\tau, \tau) \Delta_{\nu} g\left(x_{\tau}^{0}, y_{\tau}^{0}, u_{\tau}^{0}, \tau\right) \\
& +\mathrm{E} \Delta_{\nu} H_{x}^{*}\left(\psi_{\tau}, x_{\tau}^{0}, y_{\tau}^{0}, u_{\tau}^{0}, \tau\right) \Delta_{\nu} g\left(x_{\tau}^{0}, y_{\tau}^{0}, u_{\tau}^{0}, \tau\right) \\
\leq & 0 \quad \text { a.c. } ;
\end{aligned}
$$


here

$$
\begin{aligned}
& K(\tau, \theta)=\int_{\max (\tau, \theta)}^{t_{1}}\left[Q_{t \tau}^{*} H_{x x}\left(\psi_{t}, x_{t}^{0}, y_{t}^{0}, u_{t}^{0}, t\right) Q_{t \theta}+Q_{r(t) \tau}^{*} H_{y x}\left(\psi_{t}, x_{t}^{0}, y_{t}^{0}, u_{t}^{0}, t\right) Q_{t \theta}\right. \\
& +Q_{t \tau}^{*} H_{x y}\left(\psi_{t}, x_{t}^{0}, y_{t}^{0}, u_{t}^{0}, t\right) Q_{r(t) \theta} \\
& \left.+Q_{r(t) \tau}^{*} H_{y y}\left(\psi_{t}, x_{t}^{0}, y_{t}^{0}, u_{t}^{0}, t\right) Q_{r(t) \theta}\right] d t \\
& -Q_{t_{1} \tau}^{*} p_{x x}\left(x_{t_{1}}^{0}\right) Q_{t_{1} \theta} .
\end{aligned}
$$

Proof. Let $\bar{u}_{t}$ be an admissible control and let $\bar{x}_{t}$ be the corresponding trajectory of system (11)-(4). Since the random processes

$$
\left(\psi_{t}, \beta_{t}\right) \in L_{F}^{2}\left(t_{0}, t_{1} ; \mathbf{R}^{n}\right) \times L_{F}^{2}\left(t_{0}, t_{1} ; \mathbf{R}^{n \times n}\right)
$$

are the solutions of the adjoint equations (6), the expression for an increment of functional (5) gets the form as indicated below:

$$
\begin{gathered}
\Delta J\left(u^{0}\right)=-\mathrm{E} \int_{t_{0}}^{t_{1}}\left\{\Delta_{\bar{u}} H\left(\psi_{t}, x_{t}^{0}, y_{t}^{0}, u_{t}^{0}, t\right)+\Delta_{\bar{u}} H_{x}\left(\psi_{t}, x_{t}^{0}, y_{t}^{0}, u_{t}^{0}, t\right) \Delta x_{t}\right. \\
+\Delta_{\bar{u}} H_{y}\left(\psi_{t}, x_{t}^{0}, y_{t}^{0}, u_{t}^{0}, t\right) \Delta y_{t} \\
-0.5 \Delta x_{t}^{*} H_{x x}\left(\psi_{t}, x_{t}^{0}, y_{t}^{0}, u_{t}^{0}, t\right) \Delta x_{t} \\
-0.5 \Delta x_{t}^{*} H_{y x}\left(\psi_{t}, x_{t}^{0}, y_{t}^{0}, u_{t}^{0}, t\right) \Delta y_{t} \\
-0.5 \Delta y_{t}^{*} H_{x y}\left(\psi_{t}, x_{t}^{0}, y_{t}^{0}, u_{t}^{0}, t\right) \Delta x_{t} \\
\left.\quad-0.5 \Delta y_{t}^{*} H_{y y}\left(\psi_{t}, x_{t}^{0}, y_{t}^{0}, u_{t}^{0}, t\right) \Delta y_{t}\right\} d t \\
+0.5 \Delta x_{t_{1}}^{*} p_{x x}\left(x_{t_{1}}\right) \Delta x_{t_{1}}+\eta_{1},
\end{gathered}
$$

where

$$
\begin{aligned}
& \eta_{1}= \int_{0}^{1}\left[\Delta x_{t_{1}}^{*} p_{x x}\left(x_{t_{1}}^{0}+\mu \Delta x_{t_{1}}\right)-\Delta x_{t_{1}}^{*} p_{x x}\left(x_{t_{1}}^{0}\right) \Delta x_{t_{1}}\right] d \mu \\
&+\mathrm{E} \int_{t_{0}}^{t_{1}}\left\{\int _ { 0 } ^ { 1 } \left[\Delta x_{t}^{*} H_{x x}\left(\psi_{t}, x_{t}^{0}+\mu \Delta x_{t}, y_{t}^{0}+\mu \Delta y_{t}, u_{t}^{0}, t\right) \Delta x_{t}\right.\right. \\
&\left.\left.-\Delta x_{t}^{*} H_{x x}\left(\psi_{t}, x_{t}^{0}, y_{t}^{0}, u_{t}^{0}, t\right) \Delta x_{t}\right] d \mu d t\right\} \\
&+\mathrm{E} \int_{t_{0}}^{t_{1}}\left\{\int _ { 0 } ^ { 1 } \left[\Delta y_{t}^{*} H_{y x}\left(\psi_{t}, x_{t}^{0}+\mu \Delta x_{t}, y_{t}^{0}+\mu \Delta x_{t}, u_{t}^{0}, t\right) \Delta x_{t}\right.\right. \\
&\left.+\mathrm{E} \int_{t_{0}}^{t_{1}}\left\{\int_{0}^{1}\left[\Delta x_{t}^{*} H_{x y}\left(\psi_{t}, x_{t}^{0}+\mu \Delta x_{t}, y_{t}^{0}+\mu \Delta x_{t}, u_{t}^{0}, t\right) \Delta y_{t}, x_{t}^{0}, y_{t}^{0}, u_{t}^{0}, t\right) \Delta x_{t}\right] d \mu d t\right\} \\
&\left.+\mathrm{E} \int_{t_{0}}^{t_{1}}\left\{\int_{0}^{1}\left[\Delta y_{t}^{*} H_{y y}\left(\psi_{t}, x_{t}^{0}+\mu \Delta x_{t}^{*}, y_{t}^{0}+\mu \Delta x_{t}, u_{t}^{0}, t\right) \Delta y_{t}, x_{t}^{0}, y_{t}^{0}, u_{t}^{0}, t\right) \Delta y_{t}\right] d \mu d t\right\} \\
&\left.\left.-\Delta y_{t}^{*} H_{y y}\left(\psi_{t}, x_{t}^{0}, y_{t}^{0}, u_{t}^{0}, t\right) \Delta y_{t}\right] d \mu d t\right\} .
\end{aligned}
$$


Since $x_{t}(\omega)$ is a predictable random process, properties A1-A2 imply the Fubini properties for stochastic integrals in the expression for $\eta_{1}$ [8]. Consequently, increment (12) of the cost functional will be described in the following form:

$$
\begin{aligned}
\Delta J\left(u^{0}\right)= & -0.5 \int_{t_{0}}^{t_{1}} \int_{t_{0}}^{t_{1}} \Delta_{\bar{u}} g^{*}\left(x_{\tau}^{0}, y_{\tau}^{0}, u_{\tau}^{0}, \tau\right) K(\tau, s) \Delta_{\bar{u}} g\left(x_{s}^{0}, y_{s}^{0}, u_{s}^{0}, s\right) d \tau d s \\
& -\mathrm{E} \int_{t_{0}}^{t_{1}} \Delta_{\bar{u}} H\left(\psi_{t}, x_{t}^{0}, y_{t}^{0}, u_{t}^{0}, t\right) d t \\
& -\mathrm{E} \int_{t_{0}}^{t_{1}}\left(\int_{t}^{t_{1}} \Delta_{\bar{u}} H_{x}^{*}\left(\psi_{s}, x_{s}^{0}, y_{s}^{0}, u_{s}^{0}, s\right) Q_{s t} d s\right) \Delta_{\bar{u}} g\left(x_{t}^{0}, y_{t}^{0}, u_{t}^{0}, t\right) d t \\
& -\mathrm{E} \int_{t_{0}}^{t_{1}} \Delta_{\bar{u}} H_{x}^{*}\left(\psi_{t}, x_{t}^{0}, y_{t}^{0}, u_{t}^{0}, t\right) \int_{t_{0}}^{t} Q_{s t} \eta_{s} d s d t \\
& -\mathrm{E} \int_{t_{0}}^{t_{1}}\left(\int_{t-h(t)}^{t_{1}} \Delta_{\bar{u}} H_{y}^{*}\left(\psi_{s}, x_{s}^{0}, y_{s}^{0}, u_{s}^{0}, s\right) Q_{r(s) t} d s\right) \Delta_{\bar{u}} g\left(x_{t}^{0}, y_{t}^{0}, u_{t}^{0}, t\right) d t \\
& -\mathrm{E} \int_{t_{0}}^{t_{1}} \Delta_{\bar{u}} H_{y}^{*}\left(\psi_{t}, x_{t}^{0}, y_{t}^{0}, u_{t}^{0}, t\right) \int_{t_{0}}^{r(t)} Q_{r(s) t} \eta_{s} d s d t+\eta_{2} .
\end{aligned}
$$

Here

$$
\begin{aligned}
\eta_{2}=\eta_{1}+\frac{1}{2} \mathrm{E}\{ & \Delta x_{t_{1}}^{*} p_{x x}\left(x_{t_{1}}^{0}\right) \int_{t_{0}}^{t_{1}} Q_{t_{1} \tau} \eta_{\tau} d \tau \\
& +\int_{t_{0}}^{t_{1}} \int_{t_{0}}^{t_{1}} Q_{t_{1} \tau} \eta_{\tau} p_{x x}\left(x_{t_{1}}^{0}\right) Q_{t_{1} s} \Delta_{\bar{u}} g\left(x_{s}^{0}, y_{s}^{0}, u_{s}^{0}, s\right) d \tau d s \\
& -\int_{t_{0}}^{t_{1}} \Delta x_{t}^{*} H_{x x}\left(\psi_{t}, x_{t}^{0}, y_{t}^{0}, u_{t}^{0}, t\right)\left(\int_{t_{0}}^{t} Q_{t s} \eta_{s} d s\right) d t \\
& -\int_{t_{0}}^{t_{1}}\left(\int_{t_{0}}^{t} Q_{t \tau} \eta_{\tau} d \tau\right)^{*} H_{x x}\left(\psi_{t}, x_{t}^{0}, y_{t}^{0}, u_{t}^{0}, t\right) \\
& -\int_{t_{0}}^{t_{1}} \Delta y_{t}^{*} H_{x y}\left(\psi_{t}, x_{t}^{0}, y_{t}^{0}, u_{t}^{0}, t\right)\left(\int_{t_{0}}^{t} Q_{t s} \Delta_{t s} \eta_{s} d s\right) d t \\
& \left.\left.-\int_{t_{0}}^{t_{1}}\left(\int_{t_{0}}^{t-h(t)} Q_{t-h(t) \tau} \eta_{\tau} d \tau\right)^{*} H_{x y}^{0}, y_{s}^{0}, u_{s}^{0}, s\right) d s\right) d t \\
& -\int_{t_{0}}^{t_{1}} \Delta x_{t}^{*} H_{y x}\left(x_{t}^{0}, y_{t}^{0}, u_{t}^{0}, t\right) \\
& -\int_{t}^{t_{1}}\left(\int_{t_{0}}^{t} Q_{t \tau}, y_{t}^{0}, u_{t}^{0}, t\right)\left(\int_{t_{0}}^{t-h(t)} Q_{t-h(t) s} \eta_{s} d s\right) d t \\
& \times\left(\int_{t_{0}}^{t} Q_{t s} \Delta_{\bar{u}} g\left(x_{s}^{0}, y_{s}^{0}, u_{s}^{0}, s\right) d s\right) d t \\
& \\
& \\
& \\
& \\
& \\
&
\end{aligned}
$$




$$
\begin{gathered}
-\int_{t_{0}}^{t_{1}} \Delta y_{t}^{*} H_{y y}\left(\psi_{t}, x_{t}^{0}, y_{t}^{0}, u_{t}^{0}, t\right)\left(\int_{t_{0}}^{t-h(t)} Q_{t-h(t) s} \eta_{s} d s\right) d t \\
-\int_{t_{0}}^{t_{1}}\left(\int_{t_{0}}^{t-h(t)} Q_{t-h(t) \tau} \eta_{\tau} d \tau\right)^{*} H_{y y}\left(\psi_{t}, x_{t}^{0}, y_{t}^{0}, u_{t}^{0}, t\right) \\
\left.\times\left(\int_{t_{0}}^{t-h(t)} Q_{t-h(t) s} \Delta_{\bar{u}} g\left(x_{s}^{0}, y_{s}^{0}, u_{s}^{0}, s\right) d s\right) d t\right\} .
\end{gathered}
$$

Consequently we get:

$$
\begin{aligned}
\Delta J\left(u^{0}\right)= & -0.5 \int_{t_{0}}^{t_{1}} \int_{t_{0}}^{t_{1}} \Delta_{\bar{u}} g^{*}\left(x_{\tau}^{0}, y_{\tau}^{0}, u_{\tau}^{0}, \tau\right) K(\tau, s) \Delta_{\bar{u}} g\left(x_{s}^{0}, y_{s}^{0}, u_{s}^{0}, s\right) d \tau d s \\
& -\mathrm{E} \int_{t_{0}}^{t_{1}}\left(\int _ { t } ^ { t _ { 1 } } \left[\Delta_{\bar{u}} H_{x}^{*}\left(\psi_{s}, x_{s}^{0}, y_{s}^{0}, u_{s}^{0}, s\right) Q_{s t}\right.\right. \\
& \left.\left.\quad+\Delta_{\bar{u}} H_{y}^{*}\left(\psi_{s}, x_{s}^{0}, y_{s}^{0}, u_{s}^{0}, s\right) Q_{r(s) t}\right] d s\right) \Delta_{\bar{u}} g\left(x_{t}^{0}, y_{t}^{0}, u_{t}^{0}, t\right) d t \\
& -\mathrm{E} \int_{t_{0}}^{t_{1}}\left(\int _ { t } ^ { t _ { 1 } } \left[\Delta_{\bar{u}} H_{x}^{*}\left(\psi_{s}, x_{s}^{0}, y_{s}^{0}, u_{s}^{0}, s\right) Q_{s t}\right.\right. \\
& \left.\left.\quad+\Delta_{\bar{u}} H_{y}^{*}\left(\psi_{s}, x_{s}^{0}, y_{s}^{0}, u_{s}^{0}, s\right) Q_{r(s) t}\right] \eta_{s} d s\right) d t \\
& -\mathrm{E} \int_{t_{0}}^{t_{1}} \Delta_{\bar{u}} H\left(\psi_{t}, x_{t}^{0}, y_{t}^{0}, u_{t}^{0}, t\right) d t+\eta_{2} \\
\geq & 0 .
\end{aligned}
$$

Let us consider the following spike variation:

$$
\Delta u_{t}=\Delta u_{t, \varepsilon}^{\theta}= \begin{cases}0, & t \notin[\theta, \theta+\varepsilon), \varepsilon>0, \theta \in\left[t_{0}, t_{1}\right), \\ \nu-u_{t}^{0}, & t \in[\theta, \theta+\varepsilon), \nu \in L^{2}\left(\Omega, F^{\theta}, \mathrm{P} ; \mathbf{R}^{m}\right) .\end{cases}
$$

Since $\left(x_{t}^{0}, u_{t}^{0}\right)$ is a solution of problem (11) (5) and $u_{t}^{0}$ is a singular control, the expression (13) might be described in the following form:

$$
\begin{aligned}
& \Delta_{\nu} J\left(u^{0}\right)=-\frac{1}{2} \int_{\theta}^{\theta+\varepsilon} \int_{\theta}^{\theta+\varepsilon} \Delta_{\nu} g^{*}\left(x_{\tau}^{0}, y_{\tau}^{0}, u_{\tau}^{0}, \tau\right) K(\tau, s) \Delta_{\nu} g\left(x_{s}^{0}, y_{s}^{0}, u_{s}^{0}, s\right) d \tau d s \\
&-\mathrm{E} \int_{\theta}^{\theta+\varepsilon}\left(\int _ { \theta } ^ { \theta + \varepsilon } \left[\Delta_{\nu} H_{x}^{*}\left(\psi_{s}, x_{s}^{0}, y_{s}^{0}, u_{s}^{0}, s\right) Q_{s t}\right.\right. \\
&\left.\left.+\Delta_{\nu} H_{y}^{*}\left(\psi_{s}, x_{s}^{0}, y_{s}^{0}, u_{s}^{0}, s\right) Q_{r(s) t}\right] d s\right) \\
& \times \Delta_{\nu} g\left(x_{t}^{0}, y_{t}^{0}, u_{t}^{0}, t\right) d t \\
&-\mathrm{E} \int_{\theta}^{\theta+\varepsilon}\left(\int _ { \theta } ^ { t } \left[\Delta_{\nu} H_{x}^{*}\left(\psi_{s}, x_{s}^{0}, y_{s}^{0}, u_{s}^{0}, s\right) Q_{s t}\right.\right. \\
&\left.\left.+\Delta_{\nu} H_{y}^{*}\left(\psi_{s}, x_{s}^{0}, y_{s}^{0}, u_{s}^{0}, s\right) Q_{r(s) t}\right] \eta_{s} d s\right) d t+\eta_{2} \\
& \geq 0 .
\end{aligned}
$$


We will use the following lemma [6]:

Lemma 2.2. Let conditions $I-I I I$ be met. Then a.e. in $\left[t_{0}, t_{1}\right)$,

$$
\lim _{\varepsilon \rightarrow 0} \mathrm{E}\left|\frac{x_{t, \varepsilon}^{\theta}-x_{t}^{0}}{\varepsilon}\right|^{2} \leq N,
$$

where $N>0$ is a constant and $x_{t, \varepsilon}^{\theta}$ is a trajectory corresponding to the control

$$
u_{t, \varepsilon}^{\theta}=u_{t}^{\theta}+\Delta u_{t}^{\theta} .
$$

According to Lemma 2.2 we obtain:

$$
\begin{gathered}
\eta_{2}=o\left(\varepsilon^{2}\right), \\
\mathrm{E} \int_{\theta}^{\theta+\varepsilon}\left(\int_{\theta}^{t}\left[\Delta_{\nu} H_{x}^{*}\left(\psi_{s}, x_{s}^{0}, y_{s}^{0}, u_{s}^{0}, s\right) Q_{s t}+\Delta_{\nu} H_{y}^{*}\left(\psi_{s}, x_{s}^{0}, y_{s}^{0}, u_{s}^{0}, s\right) Q_{r(s) t}\right] \eta_{s} d s\right) d t \\
=o\left(\varepsilon^{2}\right) .
\end{gathered}
$$

Finally, since $\varepsilon$ is arbitrarily small, the proof of Theorem 2.2 is complete.

\section{Problem with constraint}

Now, we will consider the stochastic control problem (1)-(5) together with the constraint

$$
\mathrm{E} q\left(x_{t_{1}}\right) \in G,
$$

where $G$ is a closed convex set in $\mathbf{R}^{k}$. Additionally, we assume that the following requirement is satisfied:

IV. Function $q(x): \mathbf{R}^{n} \rightarrow \mathbf{R}^{k}$ is continuously differentiable and

$$
|q(x)|+\left|q_{x}(x)\right| \leq N(1+|x|) .
$$

Using Theorem 2.1] of [5] and the variational principle of Ekeland [7, the following theorem was proved for the stochastic optimal control problem (11)-(5) with the endpoint constraint (15).

Theorem 3.1. Let conditions $I-I V$ hold, where $\left(x_{t}^{0}, u_{t}^{0}\right)$ is a solution of problem (1) (5)), (15) and the random processes $\left(\psi_{t}, \beta_{t}\right) \in L_{F}^{2}\left(t_{0}, t_{1} ; \mathbf{R}^{n}\right) \times L_{F}^{2}\left(t_{0}, t_{1} ; \mathbf{R}^{n \times n}\right)$ are the solutions of the following adjoint system:

$$
\left\{\begin{aligned}
& d \psi_{t}=-\left[H_{x}\left(\psi_{t}, x_{t}^{0}, y_{t}^{0}, u_{t}^{0}, t\right)+\left.H_{y}\left(\psi_{z}, x_{z}^{0}, y_{z}^{0}, u_{z}^{0}, z\right)\right|_{z=s(t)} s^{\prime}(t)\right] d t+\beta_{t} d w_{t} \\
& \quad t_{0} \leq t<t_{1}-h\left(t_{1}\right) \\
& d \psi_{t}=-H_{x}\left(\psi_{t}, x_{t}^{0}, y_{t}^{0}, u_{t}^{0}, t\right) d t+\beta_{t} d w_{t}, \quad t_{1}-h\left(t_{1}\right) \leq t<t_{1} \\
& \psi_{t_{1}}=-\lambda_{0} p_{x}\left(x_{t_{1}}^{0}\right)-\lambda_{1} q_{x}\left(x_{t_{1}}^{0}\right)
\end{aligned}\right.
$$

where $\left(\lambda_{0}, \lambda_{1}\right) \in \mathbf{R}^{k+1}$ and $\lambda_{0} \leq 0, \lambda_{1}$ is a normal to the set $G$ at point $\mathrm{E} q\left(x_{t_{1}}^{0}\right)$, and $\lambda_{0}^{2}+\left|\lambda_{1}\right|^{2}=1$. Then for all $\widetilde{u} \in U$ in $\left[t_{0}, t_{1}\right)$ we have:

$$
\max _{u \in U} H\left(\psi, x_{t}^{0}, y_{t}^{0}, u, t\right)=H\left(\psi, x_{t}^{0}, y_{t}^{0}, u^{0}, t\right) \quad \text { a.c. }
$$

Now, suppose that the following requirement is also satisfied:

A4. Function $q(x): \mathbf{R}^{n} \rightarrow \mathbf{R}^{k}$ is twice continuously differentiable and

$$
|q(x)|+\left|q_{x}(x)\right| \leq N(1+|x|), \quad\left|q_{x x}(x)\right| \leq N .
$$

The following theorem provides the second order necessary condition of optimality for the stochastic control system (11)-(5), (15). 
Theorem 3.2. Let conditions A1-A4 hold, where $u_{t}^{0}$ is a singular control of problem (11) -(5), (15) and the random processes $\left(\psi_{t}, \beta_{t}\right) \in L_{F}^{2}\left(t_{0}, t_{1} ; \mathbf{R}^{n}\right) \times L_{F}^{2}\left(t_{0}, t_{1} ; \mathbf{R}^{n \times n}\right)$ are the solutions of the system (16). Then for $\forall \nu \in \bar{U}$, a.e. $\tau \in\left[t_{0}, t_{1}\right)$ is satisfied:

$$
\begin{aligned}
\mathrm{E} \Delta_{\nu} g^{*} & \left(x_{\tau}^{0}, y_{\tau}^{0}, u_{\tau}^{0}, \tau\right) K(\tau, \tau) \Delta_{\nu} g\left(x_{\tau}^{0}, y_{\tau}^{0}, u_{\tau}^{0}, \tau\right) \\
& +\mathrm{E} \Delta_{\nu} H_{x}^{*}\left(\psi_{t}, x_{\tau}^{0}, y_{\tau}^{0}, u_{\tau}^{0}, \tau\right) \Delta_{\nu} g\left(x_{\tau}^{0}, y_{\tau}^{0}, u_{\tau}^{0}, \tau\right) \\
\leq & 0 \quad \text { a.c. }
\end{aligned}
$$

here

$$
\begin{gathered}
K(\tau, \theta)=\int_{\max (\tau, \theta)}^{t_{1}}\left[Q_{t \tau}^{*} H_{x x}\left(\psi_{t}, x_{t}^{0}, y_{t}^{0}, u_{t}^{0}, t\right) Q_{t \theta}+Q_{r(t) \tau}^{*} H_{x y}\left(\psi_{t}, x_{t}^{0}, y_{t}^{0}, u_{t}^{0}, t\right) Q_{t \theta}\right. \\
+Q_{t \tau}^{*} H_{y x}\left(\psi_{t}, x_{t}^{0}, y_{t}^{0}, u_{t}^{0}, t\right) Q_{r(t) \theta} \\
\left.+Q_{r(t) \tau}^{*} H_{y y}\left(\psi_{t}, x_{t}^{0}, y_{t}^{0}, u_{t}^{0}, t\right) Q_{r(t) \theta}\right] d t \\
-Q_{t_{1} \tau}^{*} \lambda_{0} p_{x x}\left(x_{t_{1}}^{0}\right) Q_{t_{1} \theta}-Q_{t_{1} \tau}^{*} \lambda_{1} q_{x x}\left(x_{t_{1}}^{0}\right) Q_{t_{1} \theta} .
\end{gathered}
$$

Proof. For any natural $j$ let us introduce the following approximating functional:

$$
\begin{aligned}
I_{j}(u) & =S_{j}\left(\mathrm{E} p\left(x_{t_{1}}\right)+\mathrm{E} \int_{t_{0}}^{t_{1}} l\left(x_{t}, u_{t}, t\right) d t, \mathrm{E} q\left(x_{t_{1}}\right)\right) \\
& =\min _{(c, y) \in B} \sqrt{\left|c-1 / j-\mathrm{E} p\left(x_{t_{1}}\right)-\mathrm{E} \int_{t_{0}}^{t_{1}} l\left(x_{t}, u_{t}, t\right) d t\right|^{2}+\left|y-\mathrm{E} q\left(x_{t_{1}}\right)\right|^{2},}
\end{aligned}
$$

$B=\left\{(c, y): c \leq J^{0}, y \in G\right\}$, and $J^{0}$ is the minimum value of the functional in (1) - (15). $V \equiv\left(U_{\partial}, d\right)$ is a space of controls that are obtained by the following metric:

$$
d(u, \nu)=(l \otimes P)\left\{(t, \omega) \in\left[t_{0}, t_{1}\right] \times \Omega: \nu_{t} \neq u_{t}\right\} .
$$

One can show that $V$ is the complete metric space [7].

In that case, it is easy to prove the following lemma.

Lemma 3.1. Assume that conditions A1-A4 hold, $u_{t}^{n}$ is the sequence of admissible controls from $V$ and $x_{t}^{n}$ is the sequence of corresponding trajectories of the system (11)(3). If $d\left(u_{t}^{n}, u_{t}\right) \rightarrow 0, n \rightarrow \infty$, then

$$
\lim _{n \rightarrow \infty}\left\{\sup _{t_{0} \leq t \leq t_{1}} \mathrm{E}\left|x_{t}^{n}-x_{t}\right|^{2}\right\}=0
$$

where $x_{t}$ is a trajectory corresponding to an admissible control $u_{t}$.

According to the continuity of the functional $J_{j}: V \rightarrow \mathbf{R}^{n}$ and by Ekeland's variational principle we obtain the existence of control $u_{t}^{j}$ such that $d\left(u_{t}^{j}, u_{t}^{0}\right) \leq \sqrt{\varepsilon_{j}}$. Then for all $u \in V$ we have: $I_{j}\left(u^{j}\right) \leq I_{j}(u)+\sqrt{\varepsilon_{j}} d\left(u^{j}, u\right), \varepsilon_{j}=1 / j$. This inequality means that $\left(x_{t}^{j}, u_{t}^{j}\right)$ is the solution of the following problem:

$$
\begin{cases}J_{j}(u)=I_{j}(u)+\sqrt{\varepsilon_{j}} \mathrm{E} \int_{t_{0}}^{t_{1}} \delta\left(u_{t}, u_{t}^{j}\right) d t \rightarrow \min , & \\ d x_{t}=g\left(x_{t}, y_{t}, u_{t}, t\right) d t+f\left(x_{t}, y_{t}, t\right) d w_{t}, & t \in\left(t_{0}, t_{1}\right], \\ x_{t}=\Phi(t), & t \in\left[t_{0}-h\left(t_{0}\right), t_{0}\right], \\ u_{t} \in U_{\partial} & \end{cases}
$$

Function $\delta(u, \nu)$ is determined in the following way:

$$
\delta(u, \nu)= \begin{cases}0, & u=\nu \\ 1, & u \neq \nu\end{cases}
$$


Let $\left(x_{t}^{j}, u_{t}^{j}\right)$ be a solution of problem (21). Suppose that the random processes

$$
\psi_{t}^{j} \in L_{F}^{2}\left(t_{0}, t_{1} ; \mathbf{R}^{n}\right) \quad \text { and } \quad \beta_{t}^{j} \in L_{F}^{2}\left(t_{0}, t_{1} ; \mathbf{R}^{n \times n}\right)
$$

are the solutions of the following system:

$$
\left\{\begin{aligned}
d \psi_{t}^{j}= & -\left[H_{x}\left(\psi_{t}^{j}, x_{t}^{j}, y_{t}^{j}, u_{t}^{j}, t\right)+\left.H_{y}\left(\psi_{z}^{j}, x_{z}^{j}, y_{z}^{j}, u_{z}^{j}, z\right)\right|_{z=s(t)} s^{\prime}(t)\right] d t \\
& +\beta_{t}^{j} d w_{t}, \quad t_{0} \leq t \leq t_{1}-h\left(t_{1}\right), \\
d \psi_{t}^{j}= & -H_{x}\left(\psi_{t}^{j}, x_{t}^{j}, y_{t}^{j}, u_{t}^{j}, t\right) d t+\beta_{t}^{j} d w_{t}, \quad t_{1}-h\left(t_{1}\right) \leq t<t_{1}, \\
\psi_{t_{1}}^{j}= & -\lambda_{0}^{j} p_{x}\left(x_{t_{1}}^{j}\right)-\lambda_{1}^{j} q_{x}\left(x_{t_{1}}^{j}\right),
\end{aligned}\right.
$$

where nonzero $\left(\lambda_{0}^{j}, \lambda_{1}^{j}\right) \in \mathbf{R}^{k+1}$ meet the following requirement:

$$
\left(\lambda_{0}^{j}, \lambda_{1}^{j}\right)=\left(-c_{j}+1 / j+\mathrm{E} p\left(x_{t_{1}}^{j}\right)+\mathrm{E} \int_{t_{0}}^{t_{1}} l\left(x_{t}^{j}, u_{t}^{j}, t\right) d t,-y_{j}+\mathrm{E} q\left(x_{t_{1}}^{j}\right)\right) / J_{j}^{0} .
$$

Here

$$
J_{j}^{0}=\sqrt{\left|c_{j}-1 / j-\mathrm{E} p\left(x_{t_{1}}^{j}\right)-\mathrm{E} \int_{t_{0}}^{t_{1}} l\left(x_{t}^{j}, y_{t}^{j}, t\right) d t\right|^{2}+\left|y_{j}-\mathrm{E} q\left(x_{t_{1}}^{j}\right)\right|^{2}} .
$$

It was proved in the paper [6] that: $\left(\lambda_{0}^{j}, \lambda_{1}^{j}\right) \rightarrow\left(\lambda_{0}, \lambda_{1}\right)$, if $j \rightarrow \infty$.

According to convexity, function $S_{j}$ is Gato-differentiable at the point $\mathrm{E} q\left(x_{t_{1}}^{j}\right)$. For all $(c, y) \in B$, the following inequality is obtained:

$$
\left(\lambda_{0}^{j}, c-\frac{1}{j}-\mathrm{E} p\left(x_{t_{1}}^{j}\right)-\mathrm{E} \int_{t_{0}}^{t_{1}} l\left(x_{t}^{j}, u_{t}^{j}, t\right) d t+\lambda_{1}^{j}, y-\mathrm{E} q\left(x_{t_{1}}^{j}\right)\right) \leq \frac{1}{j} .
$$

Proceeding to the limit in the last inequality, we see that $\lambda_{0} \leq 0$ and $\lambda_{1}$ is a normal to the set $G$ at $\mathrm{E} q\left(x_{t_{1}}^{0}\right)$.

Let us introduce the following lemma [6].

Lemma 3.2. Let $\psi_{t}^{j}$ be a solution of system (22), and let $\psi_{t}$ be a solution of system (16). Then

$$
\mathrm{E} \int_{t_{0}}^{t_{1}}\left|\psi_{t}^{j}-\psi_{t}\right|^{2} d t+\mathrm{E} \int_{t_{0}}^{t_{1}}\left|\beta_{t}^{j}-\beta_{t}\right|^{2} d t \rightarrow 0 \quad \text { if } d\left(u_{t}^{j}, u_{t}\right) \rightarrow 0, j \rightarrow \infty .
$$

Thus, $\psi_{t}^{j} \rightarrow \psi_{t}$ in $L_{F}^{2}\left(t_{0}, t_{1} ; \mathbf{R}^{n}\right), \beta_{t}^{j} \rightarrow \beta_{t}$, if $j \rightarrow \infty$.

From Lemma 3.2 and assumptions A1-A3,

$$
H\left(\psi_{t}^{j}, x_{t}^{j}, y_{t}^{j}, u_{t}^{j}, t\right) \rightarrow H\left(\psi_{t}, x_{t}^{0}, y_{t}^{0}, u_{t}^{0}, t\right) \quad \text { in } L_{F}^{2}\left(t_{0}, t_{1} ; \mathbf{R}^{n}\right) \quad \text { if } j \rightarrow \infty .
$$

Considering the fact that

$$
-\lambda_{0}^{j} p_{x}\left(x_{t_{1}}^{j}\right)-\lambda_{1}^{j} q_{x}\left(x_{t_{1}}^{j}\right) \rightarrow-\lambda_{0} p_{x}\left(x_{t_{1}}^{0}\right)-\lambda_{1} q_{x}\left(x_{t_{1}}^{0}\right),
$$

if $j \rightarrow \infty$, and by using assumptions A3, A4, it is easy to show that:

$$
-\lambda_{0}^{j} p_{x x}\left(x_{t_{1}}^{j}\right)-\lambda_{1}^{j} q_{x x}\left(x_{t_{1}}^{j}\right) \rightarrow-\lambda_{0} p_{x x}\left(x_{t_{1}}^{0}\right)-\lambda_{1} q_{x x}\left(x_{t_{1}}^{0}\right) \quad \text { if } j \rightarrow \infty .
$$

Now, suppose that $u_{t}^{j}$ is a singular control of the problem (21). Then Theorem 3.1)implies that

$$
\begin{aligned}
& \mathrm{E} \Delta_{\nu} g^{*}\left(x_{\tau}^{l}, y_{\tau}^{l}, u_{\tau}^{l}, \tau\right) K(\tau, \tau) \Delta_{\nu} g\left(x_{\tau}^{l}, y_{\tau}^{i}, u_{\tau}^{i}, \tau\right) \\
& \quad+\mathrm{E} \Delta_{\nu} H_{x}^{*}\left(\psi_{\tau}^{i}, x_{\tau}^{i}, y_{\tau}^{i}, u_{\tau}^{i}, \tau\right) \Delta_{\nu} g\left(x_{\tau}^{i}, y_{\tau}^{i}, u_{\tau}^{i}, \tau\right)+\sqrt{\varepsilon_{i}} \int_{t_{0}}^{t_{1}} \delta\left(\nu_{t}, u_{t}^{j}\right) d t \\
& \quad \leq 0 \quad \text { a.c. }
\end{aligned}
$$


where

$$
\begin{aligned}
& K^{j}(\tau, \theta)=\int_{\max (\tau, \theta)}^{t_{1}}[ Q_{t \tau}^{j *} H_{x x}\left(\psi_{t}^{j}, x_{t}^{j}, y_{t}^{j}, u_{t}^{j}, t\right) Q_{t \theta}^{j} \\
&+Q_{r(t) \tau}^{j *} H_{x y}\left(\psi_{t}^{j}, x_{t}^{j}, y_{t}^{j}, u_{t}^{j}, t\right) Q_{t \theta}^{j} \\
&+Q_{t \tau}^{j *} H_{y x}\left(\psi_{t}^{j}, x_{t}^{j}, y_{t}^{j}, u_{t}^{j}, t\right) Q_{r(t) \theta}^{j} \\
&\left.+Q_{r(t) \tau}^{j *} H_{y y}\left(\psi_{t}^{j}, x_{t}^{j}, y_{t}^{j}, u_{t}^{j}, t\right) Q_{r(t) \theta}^{j}\right] d t \\
&-Q_{t_{1} \tau}^{j *} \lambda_{0}^{j} p_{x x}\left(x_{t_{1}}^{j}\right) Q_{t_{1} \theta}^{j}-Q_{t_{1} \tau}^{j *} \lambda_{1}^{j} q_{x x}\left(x_{t_{1}}^{0}\right) Q_{t_{1} \theta}^{j},
\end{aligned}
$$

and $Q_{t s}^{j}$ is a solution of following matrix equation:

$$
\begin{gathered}
d Q_{t \tau}^{j}=\left(g_{x}\left(x_{t}^{j}, y_{t}^{j}, u_{t}^{j}, t\right) Q_{t \tau}^{j}+g_{y}\left(x_{t}^{j}, y_{t}^{j}, u_{t}^{j}, t\right) Q_{r(t) \tau}^{j}\right) d t \\
+\left(f_{x}\left(x_{t}^{j}, y_{t}^{j}, t\right) Q_{t \tau}^{j}+f_{y}\left(x_{t}^{j}, y_{t}^{j}, t\right) Q_{r(t) \tau}^{j}\right) d w_{t}, \quad t>\tau ; \\
Q_{\tau \tau}^{j}=I ; \\
Q_{t \tau}^{j}=0, t<\tau .
\end{gathered}
$$

Using Lemma 2.2 and the assumptions A1, A2, we obtain

$$
Q_{i s}^{j} \rightarrow Q_{t s} \text { in } L_{F}^{2}\left(t_{0}, t_{1} ; \mathbf{R}^{n}\right) \quad \text { as } j \rightarrow \infty .
$$

From the assumptions A1-A4 and (23) it follows that we can proceed to the limit in (25):

$$
K^{j}(\tau, \theta) \rightarrow K(\tau, \theta)
$$

Finally, taking the limit in (24) we complete the proof of Theorem 3.2

Finally, we will consider the example given below to illustrate our theory.

Example. Consider the following optimal control problem for a stochastic system with delay:

$$
\begin{cases}d x_{1}(t)=\left(x_{1}^{2}(t)+u(t)\right) d t-2 d w_{1}(t), & t \in(0,2], \\ d x_{2}(t)=\left[x_{1}^{3}(t-1)-x_{1}^{2}(t)+u^{4}(t)\right] d t+d w_{2}(t), & t \in(0,2], \\ x_{i}(t)=0, & t \in[-1,0], i=1,2, \\ |u(t)| \leq 1, & t \in[0,2], \\ |u(t)| \leq 1, & t \in[0,2], \\ J(u)=\mathrm{E} \int_{0}^{2} x_{1}(t) \times x_{2}(t) d t \rightarrow \min .\end{cases}
$$

It is easy to show that $x_{1}(t)=0$ and $x_{2}(t)=w(t)$ is the solution of system (26) corresponding to the singular control $u(t)=0$. Now, check the condition (18). For this example,

$$
K(\theta, \theta)=\operatorname{diag}[2(2-\theta), \theta] .
$$

According to Theorem 2.2 for optimality of admissible control, $u(t)=0$ must imply that

$$
v K(\theta, \theta) v=2(2-\theta) v^{2} \leq 0 \text { for all }|v| \leq 1,0 \leq \theta \leq 2 .
$$

On the other hand, this is impossible for any $v \neq 0, \theta \neq 2$. Thus, the control $u(t)=0$ is not optimal. 


\section{BIBLIOGRAPHY}

1. V. B. Kolmanovskiŭ and A. D. Myshkis, Applied Theory of Functional-Differential Equations, Kluwer Academic Publishers, Dordrecht, 1992. MR,1256486 (95f:34092)

2. E. F. Tsar'kov, Random Perturbations of Functional-Differential Equations, Riga, 1989. (Russian) MR 1036733 (90m:34164)

3. R. Gabasov, The Qualitative Theory of Optimal Processes, Control and Systems Theory, vol. 3, M. Dekker, 1976. MR0451082 (56:9369)

4. N. I. Mahmudov and A. E. Bashirov, First order and second order necessary conditions of optimality for stochastic systems, Statistics and Control of Stochastic Processes, The Liptser Festschrift, Papers of the Steklov Seminar, Moscow 1995/96, pp. 283-295, World Sci. Publ., River Edge, NJ, 1997. MR1647306 (2000c:49048)

5. Ch. A. Agayeva and J. J. Allahverdiyeva, Maximum principle for stochastic systems with variable delay, Reports of NSA of Azerbaijan LIX (2003), 61-65. (Russian) MR2186880

6. Ch. A. Agayeva, Stochastic optimal control problem with delay, Theory of Stochastic Processes (2006), no. 1-2, 3-11. MR2316281 (2007m:93092)

7. I. Ekeland, Nonconvex minimization problem, Bull. Amer. Math. Soc. (NS) (1979), no. 1, 443474. MR.526967 (80h:49007)

8. A. E. Bashirov, Partially Observable Linear Systems under Dependent Noises, Birkhaüser, Basel, 2003. MR1960982(2004h:93002)

Yasar University, Izmir, Turkey, and Institute of Cybernetics, Baku, Azerbaijan

E-mail address: cher.agayeva@rambler.ru

E-mail address: agaeva.cherkez@yasar.edu.tr

Received 25/DEC/2007

Originally published in English 